\title{
Out-of-plane Focussing Polarization Control Grating Couplers for Photonic-Spintronic Integration
}

\author{
Hamna Becker ${ }^{1}$, Clemens J. Krïckel ${ }^{2}$, Dries Van Thourhout ${ }^{2}$, Martijn J. R. Heck ${ }^{1}$ \\ 1. Department of Engineering, Aarhus University, Denmark \\ 2. Photonics Research Group, Department of Information Technology, Ghent University - imec
}

We demonstrate the first out-of-plane 2D focusing grating coupler (FGC), designed for compact photonicspintronic integration allowing full polarization control of the emitted light. The couplers are designed for a standard $220 \mathrm{~nm}$-SOI platform and fabricated with $193 \mathrm{~nm}$ UV lithography. These couplers can find applicability as polarization (de)multiplexers, optical layer couplers or to realize optically enabled spintronic memory based on helicity dependent all-optical switching (AOS)[1,2].

In contrast to out-of-plane 1D FGCs [3], 2D designs allow additional polarization control of the emitted light. Here, in particular, we aim to illuminate optically switchable spintronic memory elements [1]. The binary state of the memory element can be changed depending on the handedness of the incident light within a given energy range [2]. The devices are designed with a 10x10 $\mathrm{mm}$ footprint, which presents outof-plane 2D FGCs with the smallest footprint published so far. To achieve focusing, the phase matching conditions are numerically solved and produce the well known photonic crystal array of 2D FGCs [4].

We evaluate the focusing characteristics experimentally by spatially scanning with a lensed fiber at multiple distances. The scan profile measures the mode overlap, which infers an approximated mode profile. The extracted effective focal spot area $\left(A_{F W H M}\right)$ and corresponding coupling efficiency are exemplarily shown in Fig. 1a. The measurements determine the focal spot location at the fiber position of $4 \mu \mathrm{m}$ and an effective focal spot area of $3.1 \mathrm{\mu m}^{2}$. This corresponds well to the simulated focal spot area of $3.3 \mathrm{um}^{2}$.

Furthermore, we thermally introduce a relative phase shift between the two optical inputs of the 2D FGC to demonstrate their full polarization control capabilities. The polarization state is analysed by means of a polarization controller. In a first approach we achieve full $2 \pi$ phase tunability with a thermal waveguide phase modulator. The polarization state is normalized and plotted for illustration on the Poincaré sphere (cf. Fig. 1b). As anticipated, this confirms that we can continuously change the state of polarization by tuning the relative phase delay of the two inputs. A second polarization control scheme relies on ring resonators (cf Fig. 1c). The ring modulator (FSR $=14.2 \mathrm{~nm}, \mathrm{FWHM}=0.6 \mathrm{~nm}, \lambda_{\text {res }}$ $=1543.8 \mathrm{~nm}, \kappa=0.4$ ) exhibits a continuous phase shift of $2 \pi$, whereas a more abrupt phase change of $\pi$ can be observed crossing the resonance as shown in Fig. 1 d). An abrupt phase change is characteristic for critical coupling [5] and poses a promising path to extremely fast and energy-efficient polarization state switching. In conclusion, we demonstrated compact out-of-plane 2D FGCs and verified their polarization controlling capabilities with different modulation schemes. Showing that these devices provide a suitable solution for photonic layer coupling and spintronic integration.

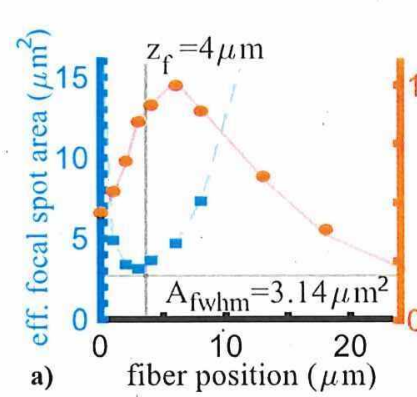

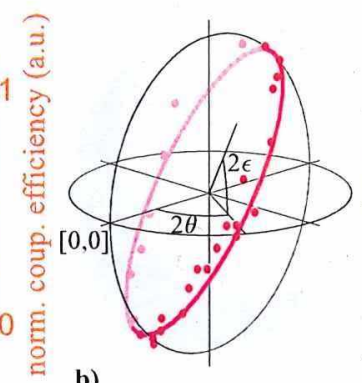

b)

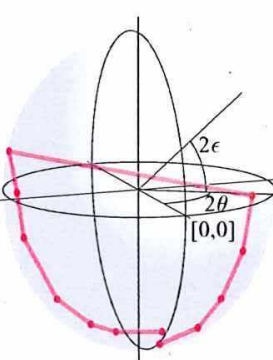

c)

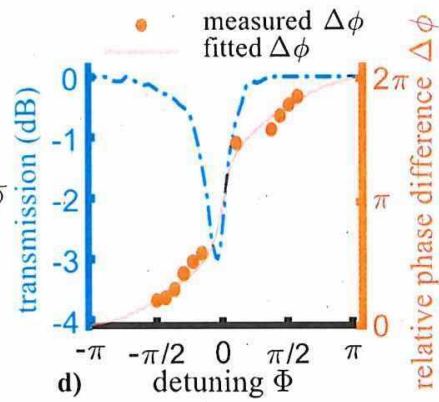

d)

Fig. 1 a) Experimental characterization of the focusing properties of the out-of-plane 2D FGCs. b) Measured polarization states produced by thermal phase tuning and fitted tunability of the polarization state illustrated on the Poincaré sphere. c) Measured polarization states produced by a ring modulator illustrated on the Poincaré sphere. d) Transmission spectrum and extracted phase difference $\Delta \phi$ across the ring resonance over the detuning $\Phi$.

This project has received funding from the European Union's Horizon 2020 research and innovation programme under grant agreement No 713481.

References

[1] Chen et al., "All-Optical Switching of Magnetic Tunnel Junctions ...," Phys. Rev. Appl. 21001, 2-7 (2017).

[2] Lalieu et al., "Integrating all-optical switching with spintronics," Nat. Commun. 10, No. 110, 1-6 (2019).

[3] Kerman et al., "Integrated Nanophotonic Excitation and Detection ...," ACS Photonics 4, No. 8, 1937-1944 (2017).

[4] Van Laere et al., "Focusing Polarization Diversity Grating Couplers ...," J. Light. Technol. 27, No. 5,'612-618 (2009).

[5] Bogaerts et al., "Silicon microring resonators," Laser Photonics Rev. 6 73, No. 1, 47-73 (2012). 
Eile Edit View History Bookmaiks Iools Heip

\begin{tabular}{|c|c|}
\hline Q Qunsic Q shanks hetter w $x$ & $\approx$ Gimil grats opstag the in $\quad X$ \\
\hline
\end{tabular}

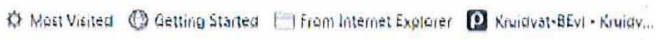

JUURNALS - PRULtEUINGS UIHERRESUURCES - MYFavorites - Recent Pages -

\section{Out-of-plane Focussing Polarization Control Grating Couplers for Photonic-Spintronic Integration}

Hanna Becker, Clemens J. Krückel, Dries Van Thourhout, and Marcijn J. R. Heck

Author information. Q Find other works by these authots.
The European Conference on Lasers and Electro. Optics 2019 Munich Germany
$23-2 T$ Juté 2019 ISEN: $979 \cdot 1 \cdot 7281 \cdot 0+5990$

from the sescion Light coupling (ck_2)

2019 Conference on Lasers and Electro. Qptics Europe and European Quantum Electronics Conlerence OSA Technical Qigeat (Optical Society oi America, 2019), papar ck.22)

\begin{tabular}{|c|c|}
\hline 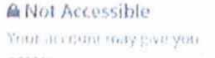 & Abstract \\
\hline & $\begin{array}{l}\text { We demonstrate the first out-of-plane } 2 D \text { focusing grating coupler (FGC), designed for compact } \\
\text { photonic-spintronic integration allowing full polarization control of the emitted light. The }\end{array}$ \\
\hline Abstract & $\begin{array}{l}\text { couplers are designed for a standard } 220 \mathrm{~nm}-501 \text { platform and fabricated with } 193 \mathrm{~nm} \text { UV } \\
\text { lithography. These couplers can find applicability as polarization (de)imultiplexers, optical layer }\end{array}$ \\
\hline References (5) & $\begin{array}{l}\text { couplers or to realize optically enabled spintronic memory based on helicity dependent atl- } \\
\text { optical switching (AOS)[1,2]. }\end{array}$ \\
\hline Back to Top & \$2 2019 IEEE \\
\hline & PDF Article \\
\hline
\end{tabular}

Xmail $\because$ Share .

" $n$ Get Citation.

回 Get PDF (743 KB),

골 Save article to My Favorites

Related Topics

Optics \& Photonics Topics

Grating couplers

Phase matching

Phase shift

Polarization

Polarization control 